\title{
CARTWRIGHT'S THEOREM ON FUNCTIONS BOUNDED AT THE INTEGERS
}

\author{
H. C. LIU AND A. J. MACINTYRE
}

1. The theorem of Cartwright [4] that for functions of exponential type not exceeding $k<\pi$ bounded at the integers

$$
\sup _{-\infty<x<+\infty}|f(x)| \leqq A(k) M, M=\sup |f(n)|, n=0, \pm 1, \pm 2, \cdots
$$

has been followed by many estimates of the value of $A(k)[1 ; 3 ; 5 ; 6]$. We shall generally mean by $A(k)$ its smallest possible value. It has been shown [3] that as $k$ approaches $\pi, A(k)$ must tend to infinity like $\log \{1 /(\pi-k)\}$. For $k$ near $\pi$ the estimates of $A(k)$ from above and below are in close agreement [5]. But for small $k$, for example $0<k<\pi / 2$ we have only $[1 ; 3]$

$$
1 \leqq A(k) \leqq 2+\frac{\pi}{3(\pi-k)} .
$$

The lower estimate can be improved. The function $f(x)=\sin (\pi x / N)$ is of type $\pi / N$ and if $N$ is an odd integer sup $|f(n)|=\cos (\pi / 2 N)$ while $\sup |f(x)|=1$. At any rate for some small values of $k$ we have

$$
A(k) \geqq \sec (k / 2) \text {. }
$$

It is a very natural conjecture that $A(k)$ tends to unity as $k$ tends to zero.

We are able to establish this conjecture in the following way.

Given the existence of $A(k)$ and an upper estimate $A_{0}(k)$ it follows from Bernstein's theorem [2, p. 206] that

$$
\left|f^{\prime}(x)\right| \leqq k A_{0}(k) M, \quad\left|f^{\prime \prime}(x)\right| \leqq k^{2} A_{0}(k) M, \cdots .
$$

Use of these inequalities in various ways leads to different estimates for $A(k)$, the best being

$$
\begin{array}{lr}
A(k) \leqq\left(1-k^{2} / 8\right)^{-1}, & \left(0<k<2^{3 / 2}\right), \\
A(k) \leqq 2 /(3-k), & (2<k<3) .
\end{array}
$$

Comparison with (1) shows that for small $k$ our estimate (3) is asymptotically correct. The upper and lower estimates are each $1+k^{2} / 8+O\left(k^{4}\right)$. For $k=\pi / 2$ we have $A(\pi / 2) \geqq 2^{1 / 2}$, which is also ob-

Presented to the Society, April 23, 1960; received by the editors April 6, 1960 and, in revised form, June 1, 1960. 
tained in [4]. The estimate (3) is numerically 1.446 which exceeds $2^{1 / 2}$ by less than 3 per cent.

2. If the inequality (2) is integrated between $x$ and the nearest integer $n$ we have

$$
|f(x)-f(n)| \leqq|x-n| k A_{0}(k) M \leqq k A_{0}(k) M / 2
$$

and hence

$$
|f(x)| \leqq\left\{1+k A_{0}(k) / 2\right\} M .
$$

The new constant $1+k A_{0}(k) / 2$ will be less than $A_{0}(k)$ if $k<2$ and

$$
A_{0}(k)>1+k A(k) / 2 \text { or } A_{0}(k)>2 /(2-k) .
$$

We can infer that

$$
|f(x)| \leqq 2 M /(2-k)
$$

by an iterative argument. Set

$$
A_{1}(k)=1+k A_{0}(k) / 2, \quad A_{n+1}(k)=1+k A_{n}(k) / 2 .
$$

Evidently

$$
|f(x)| \leqq A_{n}(k) M=\left\{2 /(2-k)+(k / 2)^{n}\left[A_{0}(k)-2 /(2-k)\right]\right\} M .
$$

Since $n$ can be arbitrarily large,

$$
|f(x)| \leqq 2 M /(2-k) .
$$

3. This simple argument is sufficient to establish the conjecture that $A(k)$ tends to unity as $k$ tends zero. ${ }^{1} \mathrm{~A}$ slight improvement is obtained by using a variant of Bernstein's theorem [2, p. 214] namely that if $f(z)$ is an entire function of exponential type $k$ bounded on the real axis then for $0<2 \delta<\pi / k$

$$
|f(t+\delta)-f(t-\delta)| \leqq 2 \sin (\delta k) \sup |f(x)| .
$$

If this inequality is used with $x=t \pm \delta$ and $t \mp \delta$ the nearest integer, in place of (5) then $\delta \leqq 1 / 4$ and

$$
\sup |f(x)| \leqq M+2 \sin (k / 4) A_{0}(k) M .
$$

Arguing as before we now infer that

$$
\sup |f(x)| \leqq\{1-2 \sin (k / 4)\}^{-1} \sup |f(x)|,
$$

this inequality being valid for $0<k<2 \pi / 3$.

${ }^{1}$ R. P. Boas, Jr. gives us another proof. Suppose $p k<\pi, p$ is an integer; then $f(z)^{p}$ is of type $p k$ and $|f(x)| p \leqq M^{p}$. So $|f(x)|^{p} \leqq A(p k) M^{p},|f(x)| \leqq A(p k)^{1 / p} M$. 
4. Lagrange's interpolation formula [7]

$$
f(x)=\frac{x-b}{a-b} f(a)+\frac{x-a}{b-a} f(b)+\lambda \frac{(x-a)(x-b)}{2} f^{\prime \prime}(\xi),|\lambda| \leqq 1,
$$

may also be used with $a=n<x<b=n+1$.

Since $\left|f^{\prime \prime}(x)\right| \leqq k^{2} A_{0}(k) M$ by repeated use of Bernstein's theorem, we have

$$
|f(x)| \leqq M+(x-a)(b-x) k^{2} A_{0}(k) M / 2 \leqq M+k^{2} A_{0}(k) M / 8 .
$$

This leads to the estimate

$$
\sup |f(x)| \leqq\left(1-k^{2} / 8\right)^{-1} \sup |f(n)|
$$

valid for $k<2^{3 / 2}$.

It may be noted that if $k>2$ our use of Lagrange's interpolation is inferior to the more elementary inequality

$$
|f(x)-f(n)| \leqq|x-n| k A_{0}(k) M
$$

when $|x-n|<1 / 2-1 / k$. If we use (7) in the intervals $n \leqq x \leqq n+1 / 2$ $-1 / k, n+1-(1 / 2-1 / k) \leqq x \leqq n x+1$ and (6) for $a \leqq x \leqq b$ with $a=n$ $+1 / 2-1 / k$ and $b=n+1-(1 / 2-1 / k)$, we evidently obtain

$$
|f(x)| \leqq M+(1 / 2-1 / k) k A_{0}(k) M+k^{-2} k^{2} A_{0}(k) M / 2 .
$$

This leads to the inequality

$$
\sup |f(x)| \leqq \frac{2}{3-k} \sup |f(n)|
$$

valid for $2<k<3$.

\section{REFERENCES}

1. R. P. Boas, Jr., Entire functions bounded on a line, Duke Math. J. vol. 6 (1940) pp. 148-160.

2. - Entire functions, New York, Academic Press, 1954.

3. R. P. Boas, Jr. and A. C. Schaeffer, $A$ theorem of Cartwright, Duke Math. J. vol. 9 (1942) pp. 879-883.

4. M. L. Cartwright, On certain integral functions of order one, Quart. J. Math. Oxford Ser. no. 1 vol. 7 (1936) pp. 46-55.

5. H. C. Liu, Entire functions bounded at integers, to appear in J. Indian Math. Soc.

6. A. J. Macintyre, Laplace's transformation and integral functions, Proc. London Math. Soc. (2) vol. 45 (1938-1939) pp. 1-20.

7. N. E. Nörlund, Leçons sur les sêries d'interpolation, Paris, Gauthier-Villars, 1926, p. 4, (16) p. 9.

University of Cincinnati 